\title{
Non Kählerian surfaces with a cycle of rational curves
}

https://doi.org/10.1515/coma-2020-0114

Received March 22, 2021; accepted June 20, 2021

Abstract: Let $S$ be a compact complex surface in class $\mathrm{VII}_{0}^{+}$containing a cycle of rational curves $C=\sum D_{j}$. Let $D=C+A$ be the maximal connected divisor containing $C$. If there is another connected component of curves $C^{\prime}$ then $C^{\prime}$ is a cycle of rational curves, $A=0$ and $S$ is a Inoue-Hirzebruch surface. If there is only one connected component $D$ then each connected component $A_{i}$ of $A$ is a chain of rational curves which intersects a curve $D_{j}$ of the cycle and for each curve $D_{j}$ of the cycle there at most one chain which meets $D_{j}$. In other words, we do not prove the existence of curves other those of the cycle $C$, but if some other curves exist the maximal divisor looks like the maximal divisor of a Kato surface with perhaps missing curves. The proof of this topological result is an application of Donaldson theorem on trivialization of the intersection form and of deformation theory. We apply this result to show that a twisted logarithmic 1 -form has a trivial vanishing divisor.

Keywords: class VII, Kodaira classification, compact non-Kähler surface

MSC: 32J15, 32J27

\section{Introduction}

A minimal compact complex surface $S$ is said to be of the class $\mathrm{VII}_{0}$ of Kodaira if the first Betti number satisfies $b_{1}(S)=1$ and Kodaira dimension satisfies $k(S)<0$. A surface $S$ is of class $\mathrm{VII}_{0}^{+}$if moreover $n:=b_{2}(S)>0$; these surfaces admit no nonconstant meromorphic functions. The cupproduct form $Q$ defines on $H^{2}(S, \mathbb{R})$ a negative definite form and in 1987 S.K. Donaldson proved

Theorem 1. 1 ([6]). If $X$ is closed, oriented smooth 4-manifold whose intersection form

$$
Q: H^{2}(S, \mathbb{Z}) / \text { Torsion } \rightarrow \mathbb{Z}
$$

is negative definite, then the form is equivalent over the integers to the standard form $(-1) \oplus(-1) \oplus \cdots \oplus(-1)$.

In other words, if $n=b_{2}(S)$, there are classes $e_{0}, \ldots, e_{n-1}$ such that $e_{i} \cdot e_{j}=-\delta_{i j}$. Under the further assumption that $S$ contains a cycle of rational curves, I. Nakamura [10, Thm 1.5] proved the existence of these classes by deformation theory. In fact, $S$ can be deformed into a blown-up Hopf surface $n$ times [10] and the exceptional curves (or divisors) give the requested classes. All known surfaces in class $\mathrm{VII}_{0}^{+}$are Kato surfaces i.e. contain Global Spherical Shells (GSS). The major problem in the classification of non-Kählerian surfaces is to achieve the classification of surfaces $S$ of class $\mathrm{VII}_{0}^{+}$. Are all surfaces in class $\mathrm{VII}_{0}^{+}$Kato surfaces ? Till now, the answer is positive for $b_{2}(S)=1$ by A. Teleman [12]. By definition a GSS is a biholomorphic map $\varphi: U \rightarrow V$ from a neighbourhood $U \subset \mathbb{C}^{2} \backslash\{0\}$ of the sphere $S^{3}=\partial B^{2}$ onto an open set $V$ such that $\Sigma=\varphi\left(S^{3}\right)$ does not disconnect $S$. By construction, rational curves are obtained as strict transform of exceptional curves. If for a subset $I \subset\{0, \ldots, n-1\}$ we denote $e_{I}:=\sum_{i \in I} e_{i}$, we can expect that the class of a curve is of the type $e_{i}-e_{I}$,

^Corresponding Author: Georges Dloussky: Aix Marseille Université, CNRS, Centrale Marseille, I2M, UMR 7373, 13453, Marseille, France, E-mail: georges.dloussky@univ-amu.fr 
$i \notin I$. In [4] the following theorem is proved under, at one step, the existence of a Numerically AntiCanonical (NAC) divisor. In this article we avoid this assumption. Odd Inoue-Hirzebruch surfaces contain exactly one cycle are also called half Inoue-Hirzebruch surface. See [3] for a construction by contracting germs.

Theorem 1. 2. Let $S$ be a minimal surface in class VII ${ }_{0}^{+}$with a cycle

$$
C=D_{0}+\cdots+D_{s-1}=-\left(e_{r}+\cdots+e_{n-1}\right)
$$

of $s \geq 1$ rational curves.

1) $S$ is an odd Inoue-Hirzebruch surface if and only if $\left[H_{1}(C, \mathbb{Z}): H_{1}(S, \mathbb{Z})\right]=2$. In this case $s=n, r=0$ and

$$
\sharp(C)-C^{2}=2 b_{2}(S) .
$$

2) If $H_{1}(C, \mathbb{Z})=H_{1}(S, \mathbb{Z})$, then $s=r$,

$$
\sharp(C)-C^{2}=b_{2}(S),
$$

all curves are of type $\mathbf{a}$ i.e. $D_{i}=e_{i}-e_{I_{i}}, i \notin I_{i}$, and $\left(I_{i}\right)_{0 \leq i \leq s-1}$ is a partition of $[0, n-1]$.

By this theorem it is possible to detect which exceptional curve of the first kind appears in a deformation of $S$ where one singular point of the cycle is smoothed (see theorem 18). Since a surface with a cycle can be deformed into a blown-up primary Hopf surface [10] the groups $H_{2}(S, \mathbb{Z})$ and $H^{2}(S, \mathbb{Z})$ have no torsion.

By simple computations on the Donaldson classes it is easy to prove (see thm 19):

Theorem 1. 3. Let $C=D_{0}+\cdots+D_{\alpha_{i}}+\cdots+D_{\alpha_{s-1}}$ be a cycle of $1 \leq s<n$ rational curves, $D=C+A$ be the maximal connected reduced divisor containing $C$. Then there is a subset $\mathfrak{D} \subset[0, n-1]$, such that

$$
D=C+A=-e_{\mathfrak{D}} \in H_{2}(S, \mathbb{Z}) .
$$

If $A$ is not empty, each connected component $A_{i}$ of $A$ called a tree is a chain of rational curves. Moreover two trees $A_{j}, A_{k}$ meet different curves of the cycle.

In a series of papers [12], [13], [14], A. Teleman developped a strategy using gauge theory, successfull for $b_{2}(S) \leq 3$, to show that any surface $S$ in class $\mathrm{VII}_{0}^{+}$contains a cycle of rational curves. The aim is to prove that a class of type $-e_{I}$ is represented by a one dimensional analytic subspace $C$. It is easy to show that with this condition $C$ has to contain a cycle. Theorem 3 gives a reason (among others) why it is difficult to show the existence of the missing curves even when $b_{2}(S)=2$. Recall that if there are $b_{2}(S)$ rational curves the surface is Kato [5]. Finally we obtain, with deformation theory, the following result which show with theorem 1.3 that the maximal divisor looks like the one of a Kato surface (see [2] or [9]).

Theorem 1. 4. Let $S$ be a surface in class VII ${ }_{0}^{+}$containing a cycle $C$ of $s \geq 1$ rational curves and $D=C+A$ the connected component of the cycle. If there is another connected component $C^{\prime}$ of curves, $C^{\prime}$ is a cycle of rational curves, $A=0$ and $S$ is a Inoue-Hirzebruch surface.

We apply this result to show that a twisted logarithmic 1-form on a surface in class $\mathrm{VII}_{0}^{+}$cannot vanish along a divisor.

\section{Topological classes of curves and chains}

One supposes that $S$ contains a cycle of $s$ rational curves, $1 \leq s \leq n$. When $s=1$ it is a rational curve with a double point, when $s=2$ it is the union of two rational curves $D_{0}, D_{1}$ such that $D_{0} . D_{1}=2$, and when $s \geq 3$, $D_{0} . D_{1}=D_{1} \cdot D_{2}=\cdots=D_{s-1} \cdot D_{0}=1$.

Since a surface in class $\mathrm{VII}_{0}^{+}$with a cycle of rational curves can be deformed into a blown-up Hopf surface, 
the groups $H^{2}(S, \mathbb{Z})$ and $H_{2}(S, \mathbb{Z})$ have no torsion. By Poincaré duality they are isomorphic, therefore we shall denote in the same way a class $e_{i}$ in $H^{2}(S, \mathbb{Z})$ and its dual in $H_{2}(S, \mathbb{Z})$.

We recall basic facts used in [10], [4] and in the next sections. For the sake of completeness we give a proof:

Lemma 2.5 ([10] (2.5), (2.6), (2.7)). 1) Let $D$ be a nonsingular rational curve. If $D=a_{0} e_{0}+\cdots+a_{n-1} e_{n-1} \in$ $\mathrm{H}_{2}(S, \mathbb{Z})$, then there exists a unique $i \in[0, n-1]$ such that $a_{i}=1$ or -2 , and $a_{j}=0$ or -1 for $j \neq i$.

2) Let $D_{1}$ and $D_{2}$ two distinct divisors without common irreducible component such that $D_{1}=e_{i_{1}}-e_{I_{1}}, i_{1} \notin I_{1}$ and $D_{2}=e_{i_{2}}-e_{I_{2}}, i_{2} \notin I_{2}$, then $i_{1} \neq i_{2}$.

Proof: 1) For a rational curve we have $0=K D+D^{2}+2=2-\sum_{k}\left(a_{k}^{2}+a_{k}\right)$, therefore $a_{i}^{2}+a_{i}=2$ for a unique index and $a_{k}^{2}+a_{k}=0$ for the others.

2) If $i_{1}=i_{2},\left(e_{i_{1}}-e_{I_{1}}\right) \cdot\left(e_{i_{2}}-e_{I_{2}}\right)=-1+e_{I_{1}} \cdot e_{I_{2}}<0$ which is impossible.

Lemma 2.6 ([10] (2.4)). Let $S$ be a surface of class VII $I_{0}^{+}$with a cycle $C=D_{0}+\cdots+D_{s-1}=-\left(e_{r}+\cdots+e_{n-1}\right)$ of rational curves and without divisor $D$ such that $D^{2}=0$. Let $L_{I}:=\bigotimes_{i \in I} L_{i}, L=L_{I} \otimes F, F \in H^{1}\left(S, \mathbb{C}^{\star}\right)$ for any subset $I \subset[0, n-1]$. Then we have:

i) If $I \neq[0, n-1]$, then $H^{q}(S, L)=0$ for any $q$.

ii) If $L \otimes \mathcal{O}_{C}=\mathcal{O}_{C}$, then $I=[0, r-1]$, and $F=\mathcal{O}_{S}, K_{S} \otimes L^{*} \otimes[C]=\mathcal{O}_{S}$.

iii) If $L D_{i}=0$ for any irreducible component $D_{i}$ of $C$, then $I=[0, r-1]$.

Let $S$ be a surface containing a cycle $C=D_{0}+\cdots+D_{s-1}$ of $s$ rational curves. Lemma 2.5 shows that in the cycle there are two possible types of nonsingular rational curves:

- (Type a) $D_{i}=e_{i}-e_{I_{i}}$ with $i \notin I_{i}$,

- (Type b) $D_{i}=-2 e_{i}-e_{I_{i}}$ with $i \notin I_{i}$.

we shall show that the same dichotomy applies for chains of nonsingular rational curves.

Lemma 2.7. Let $D^{\prime}=D_{j}+\cdots+D_{j+p-1}$ be a chain of $p \geq 1$ rational curves contained in $C$ (where the indices are taken modulo s). Then $D^{\prime}$ is of type $\mathbf{a}$ or of type $\mathbf{b}$ and $D^{\prime}$ is of type $\mathbf{b}$ if $D^{\prime}$ contains a subchain of type $\mathbf{b}$. In particular a cycle cannot contain two disjoint chains of type $\mathbf{b}$.

Proof: On induction on $p \geq 1$, the case $p=1$ being proved in Lemma 2.5. Let $D^{\prime}=D_{j}+\cdots+D_{j+p}$ be a chain of $p+1$ rational curves contained in $C$. By induction hypothesis $D^{\prime \prime}=D_{j}+\cdots+D_{j+p-1}$ is of type $\mathbf{a}$ or $\mathbf{b}$ and we have to consider $D^{\prime}=D^{\prime \prime}+D_{j+p}$. Since

$$
\left(-2 e_{i}-e_{I}\right)\left(-2 e_{j}-e_{J}\right) \leq 0
$$

not both are of type $\mathbf{b}$.

- If one, say $D^{\prime \prime}$ is of type $\mathbf{b}$, we have in $H_{2}(S, \mathbb{Z})$,

$$
D^{\prime \prime}=-2 e_{i}-e_{I}, \quad D_{j+p}=e_{j}-e_{J}
$$

with $i \notin I, j \notin J$. Therefore,

$$
1=\left(-2 e_{i}-e_{I}\right)\left(e_{j}-e_{J}\right)=-2 e_{i} e_{j}-e_{j} e_{I}+2 e_{i} e_{J}+e_{I} e_{J}
$$

and

- either $j \in I, i \neq j, i \notin J, I \cap J=\emptyset$, whence

$$
D^{\prime \prime}+D_{j+p}=-2 e_{i}-e_{I}+e_{j}-e_{J}=-2 e_{i}-e_{I \cup J \backslash\{j\}}
$$

is of type $\mathbf{b}$, 
- or $j \notin I, i=j, i \notin J$ and $I \cap J$ contains one element, say $k$. Setting $I^{\prime}=I \backslash\{k\}$ and $J^{\prime}=J \backslash\{k\}$ we obtain

$$
D^{\prime \prime}+D_{j+p}=-2 e_{k}-e_{\{i\} \cup I^{\prime} \cup J^{\prime}}
$$

is of type $\mathbf{b}$.

- Both are of type $\mathbf{a}$

$$
D^{\prime \prime}=e_{i}-e_{I}, \quad D_{j+p}=e_{j}-e_{J}
$$

with $i \notin I, j \notin J$. Clearly $i \neq j$ and

$$
1=D^{\prime \prime} D_{j+p}=-e_{i} e_{J}-e_{j} e_{I}+e_{I} e_{J},
$$

then $i \in J$ or $j \in I$, say $j \in I$.

If $I \cap J=\emptyset, i \notin J$, we set $I^{\prime}=I \backslash\{j\}$ and $D^{\prime \prime}+D_{j+p}=e_{i}-e_{I^{\prime} \cup J}$ is of type a.

If $I \cap J=\{k\}, i \in J$ and we set $I^{\prime}=I \backslash\{j, k\}, J^{\prime}=J \backslash\{i, k\}$. Therefore

$$
D^{\prime \prime}+D_{j+p}=-2 e_{k}-e_{I^{\prime} \cup J^{\prime}}
$$

is of type $\mathbf{b}$.

In the following examples we use notations used in [2], $a(S)=\left(a_{i}\right)_{i \in \mathbb{Z}}$ is the sequence of (opposite of) selfintersections of the rational curves in the universal covering space $\tilde{S}$ of $S$. This sequence is periodic of period $n$ and we overline a period.

Examples 2. 8. If $a(S)=(\overline{333})$ then we obtain a cycle $C=D_{0}+D_{1}+D_{2}$,

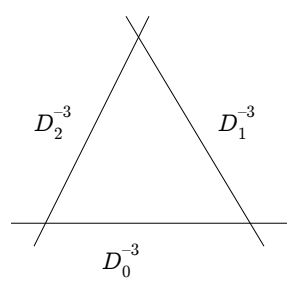

All the curves are of type a: $D_{0}=e_{0}-\left(e_{1}+e_{2}\right), D_{1}=e_{1}-\left(e_{2}+e_{0}\right), D_{2}=e_{2}-\left(e_{0}+e_{1}\right), C=-\left(e_{0}+e_{1}+e_{2}\right)$, however the chain $D_{0}+D_{1}=-2 e_{2}$ is of type $\mathbf{b}$ with $I$ empty.

The previous example 2.8 is generalized in the following lemma:

Lemma 2. 9. Let $D^{\prime}=D_{0}+\cdots+D_{j-1}$ be a chain of $j \geq 2$ rational curves of type $\mathbf{a}$. The following two conditions are equivalent:

i) $D^{\prime}$ is of type $\mathbf{b}$ and all strict subchains are of type $\mathbf{a}$.

ii) $I_{0} \cap I_{j-1}=\{k\}$ with $k \notin[0, j-1]$ and for any $0 \leq p<q \leq j-1,(p, q) \neq(0, j-1)$ we have $I_{p} \cap I_{q}=\emptyset$.

Proof: Denote $D_{i}=e_{i}-e_{I_{i}}$.

If $j=2, D^{\prime}=D_{0}+D_{1}=e_{0}-e_{I_{0}}+e_{1}-e_{I_{1}}$ is of type $\mathbf{b}$, then $I_{0} \cap I_{1}=\{k\}$ with $k \geq 2$. Conversely, if $I_{0} \cap I_{1}=\{k\}$ with $k \geq 2$, then $D_{0}+D_{1}$ is of type $\mathbf{b}$.

Suppose that $j \geq 2$ and let $D^{\prime}=D_{0}+\cdots+D_{j}=A+D_{j}$ be a chain of $j+1$ curves of type a with $A=D_{0}+\cdots+D_{j-1}$. If $\mathrm{i}$ ) is satisfied, then since all subchains are of type $\mathbf{a}, A=e_{0}-e_{I_{A}}$, with $I_{p} \cap I_{q}=\emptyset$ when $0 \leq p<q \leq j-1$. Moreover since $A+D_{j}$ is of type $\mathbf{b}$, then $I_{A} \cap I_{j}=\{k\}$, in particular $k \in I_{j}$ with $k>j$. Consider the subchain $D_{1}+\cdots+D_{j}$ which is of type a as subchain. Then for $1 \leq p<q \leq j, I_{p} \cap I_{j}=\emptyset$. Therefore $I_{0} \cap I_{j}=\{k\}$ with $k>j$.

Conversely if ii) is satisfied, subchains are of type a by induction. If $I_{0} \cap I_{j}=\{k\}$ with $k>j$, then $D^{\prime}=-2 e_{k}-e_{K}$. 
Notice that the example 2.8 is not a counterexample of the following lemma since the known example is an odd Inoue-Hirzebruch surfaces (= half-Inoue surface) with $a(S)=\left(\overline{s_{1} s_{1} s_{1}}\right)=(\overline{333})$. In this case $\left[H_{1}(S, \mathbb{Z})\right.$ : $\left.H_{1}(C, \mathbb{Z})\right]=2$.

Lemma 2. 10. Let $C=D_{0}+\cdots+D_{s-1}$ be a cycle of $s \geq 2$ rational curves, with $D_{i}=e_{i}-e_{I_{i}}, i=0, \ldots, s-1$. If $H_{1}(C, \mathbb{Z})=H_{1}(S, \mathbb{Z})$, then

$$
\forall i, j, i \neq j \Rightarrow I_{i} \cap I_{j}=\emptyset \text {. }
$$

Proof: Let $p: S^{\prime} \rightarrow S$ be a 2-sheeted covering of $S$. By assumption $C^{\prime}=p^{\star}(C)$ is a cycle $C^{\prime}$ with $2 s$ rational curves

$$
C^{\prime}=D_{0}^{\prime}+\cdots+D_{s-1}^{\prime}+D_{n}^{\prime}+\cdots+D_{n+s-1}^{\prime}
$$

with $D_{0}^{\prime} D_{1}^{\prime}=\ldots=D_{s-1}^{\prime} D_{n}^{\prime}=\ldots=D_{n+s-1}^{\prime} D_{0}^{\prime}=1$. Suppose that $I_{i} \cap I_{j}=\{k\}$. Denoting $e_{0}^{\prime}, \ldots, e_{2 n-1}^{\prime}$ the $2 n$ Donaldson classes which trivialize the intersection form, we have $p^{\star} e_{k}=e_{k}^{\prime}+e_{n+k}^{\prime}$,

$$
\begin{aligned}
& p^{\star}\left(D_{i}\right)=D_{i}^{\prime}+D_{n+i}^{\prime}=\left(e_{i}^{\prime}-e_{I_{i}}^{\prime}\right)+\left(e_{n+i}^{\prime}-e_{n+I_{i}}^{\prime}\right), \\
& p^{\star}\left(D_{j}\right)=D_{j}^{\prime}+D_{n+j}^{\prime}=\left(e_{j}^{\prime}-e_{I_{j}}^{\prime}\right)+\left(e_{n+j}^{\prime}-e_{n+I_{j}}^{\prime}\right) .
\end{aligned}
$$

If $I_{i}^{\prime} \cap I_{j}^{\prime}=\{k\}$ then $I_{n+i}^{\prime} \cap I_{n+j}^{\prime}=\{n+k\}$. By Lemma 2.9 we should obtain two disjoint chains of type $\mathbf{b}$ and this is impossible by Lemma 2.7 .

Lemma 2. 11. Let $S$ be a minimal surface with a cycle

$$
C=D_{0}+\cdots+D_{s-1}=-\left(e_{r}+\cdots+e_{n-1}\right)
$$

of $s \geq 2$ rational curves. Suppose that $H_{1}(C, \mathbb{Z})=H_{1}(S, \mathbb{Z})$ and all curves are of type a. Then $s=r$, i.e.

$$
\sharp(C)-C^{2}=b_{2}(S)
$$

and $\left(I_{i}\right)_{0 \leq s-1}$ is a partition of $[0, n-1]$.

Proof: 1) If $\boldsymbol{s}=\mathbf{2}, D_{0}=e_{i_{0}}-e_{I_{0}}, D_{1}=e_{i_{1}}-e_{I_{1}}$. We have

$$
2=D_{0} D_{1}=-e_{i_{0}} e_{I_{1}}-e_{i_{1}} e_{I_{0}}+e_{I_{0}} e_{I_{1}}
$$

whence $i_{1} \in I_{0}, i_{0} \in I_{1}, I_{0} \cap I_{1}=\emptyset$. Setting $I_{0}^{\prime}=I_{0} \backslash\left\{i_{1}\right\}$ and $I_{1}^{\prime}=I_{1} \backslash\left\{i_{0}\right\}$, we obtain

$$
D_{0}=e_{i_{0}}-e_{i_{1}}-e_{I_{0}^{\prime}}, \quad D_{1}=e_{i_{1}}-e_{i_{0}}-e_{I_{1}^{\prime}}, \quad \text { with } I_{0}^{\prime} \cap I_{1}^{\prime}=\emptyset, \quad\left\{i_{0}, i_{1}\right\} \cap\left(I_{0}^{\prime} \cup I_{1}^{\prime}\right)=\emptyset .
$$

Therefore

$$
-\left(e_{r}+\cdots+e_{n-1}\right)=C=D_{0}+D_{1}=-\left(e_{I_{0}^{\prime}}+e_{I_{1}^{\prime}}\right)
$$

i.e. $I_{0}^{\prime} \cup I_{1}^{\prime}=[r, n-1]$. Let $I=\left\{i_{0}, i_{1}\right\} \cup I_{0}^{\prime} \cup I_{1}^{\prime}$ and $I^{\prime}=[0, n-1] \backslash I$. Suppose that $I^{\prime}$ is non empty. Of course, $e_{I^{\prime}} \cdot D_{0}=e_{I^{\prime}} \cdot D_{1}=0$, whence by lemma $\left.2.6 \mathrm{iii}\right), I^{\prime}=[0, r-1]$ which is impossible. Therefore $I^{\prime}=\emptyset$ and $I_{0} \cup I_{1}=\left\{i_{0}, i_{1}\right\} \cup I_{0}^{\prime} \cup I_{1}^{\prime}=[0, n-1]$, i.e. $r=2$ and

$$
\sharp(C)-C^{2}=2+(n-2)=b_{2}(S) .
$$

2) If $s \geq 3, D_{j}=e_{i_{j}}-e_{I_{j}}, j=0, \ldots, s-1$ and we may suppose that the numbering is such that

$$
D_{0} D_{1}=\ldots=D_{s-2} D_{s-1}=D_{s-1} D_{0}=1
$$

By Lemma 2.10, $I_{i} \cap I_{j}=\emptyset$ if $i \neq j$. Moreover the equality

$$
1=D_{j} D_{j+1}=-e_{i_{j}} e_{I_{j+1}}-e_{i_{j+1}} e_{I_{j}}
$$


implies that $i_{j} \in I_{j+1}$ or $i_{j+1} \in I_{j}$.

Changing if necessary the numbering (i.e. we number the curves following the other orientation of the cycle) we suppose that $i_{1} \in I_{0}$, then a straightforward proof by induction shows that $i_{j+1} \in I_{j}$ for $j \geq 0$.

Setting $I_{j}^{\prime}=I_{j} \backslash\left\{i_{j+1}\right\}$, we have

$$
\begin{aligned}
-\left(e_{r}+\cdots+e_{n-1}\right) & =C=D_{0}+\cdots+D_{s-1}=\sum_{j=0}^{s-1}\left(e_{i_{j}}-e_{I_{j}}\right) \\
& =-\left(e_{I_{0}^{\prime}}+\cdots+e_{I_{s-1}^{\prime}}\right)
\end{aligned}
$$

therefore

$$
[r, n-1]=I_{0}^{\prime} \cup \cdots \cup I_{s-1}^{\prime} \quad \text { with } \quad\left\{i_{0}, \ldots, i_{s-1}\right\} \cap\left(I_{0}^{\prime} \cup \cdots \cup I_{s-1}^{\prime}\right)=\emptyset
$$

Setting $I=\left\{i_{0}, \ldots, i_{s-1}\right\} \cup I_{0}^{\prime} \cup \cdots \cup I_{s-1}^{\prime}$ and $I^{\prime}=[0, n-1] \backslash I$, we have $L_{I^{\prime}} D_{j}=0$ for all $j=0, \ldots, s-1$, then if $I^{\prime} \neq \emptyset$, lemma 2.6 would imply once again that $I^{\prime}=[0, r-1]$ and this is impossible, hence $I^{\prime}=\emptyset$ and $\left\{i_{0}, \ldots, i_{s-1}\right\} \cup I_{0}^{\prime} \cup \cdots \cup I_{s-1}^{\prime}=[0, n-1]$, i.e. $r=s$ and $\left\{i_{0}, \ldots, i_{r-1}\right\}=[0, r-1]$. Finally

$$
\sharp(C)-C^{2}=s+(n-r)=n=b_{2}(S) .
$$

We determine now the surfaces for which there exists a rational curve of type $\mathbf{b}$. Following notations of [2], $s_{n}$ is the sequence of $n$ integers $s_{n}=(n+2,2, \ldots, 2)$.

Lemma 2. 12. Let $S$ be a minimal surface with a cycle $C=D_{0}+\cdots+D_{s-1}=-\left(e_{r}+\cdots+e_{n-1}\right)$ of $s \geq 2$ rational curves. If there exists a rational curve of type $\mathbf{b}$, say $D_{0}$, then $s=n, r=0$,

$$
D_{0}=-2 e_{1}-e_{[2, n-1]}, \quad D_{1}=e_{1}-e_{2}, \ldots, D_{n-1}=e_{n-1}-e_{0},
$$

$S$ is the odd Inoue-Hirzebruch surface such that $a(S)=\left(\overrightarrow{s_{n}}\right)$. In particular $\left[H_{1}(C, \mathbb{Z}): H_{1}(S, \mathbb{Z})\right]=2$.

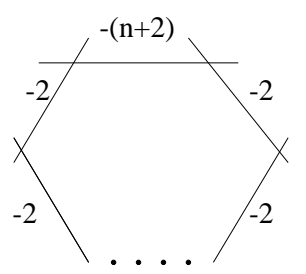

Proof: First we prove that $\left[H_{1}(C, \mathbb{Z}): H_{1}(S, \mathbb{Z})\right]=2$ by contradiction. Suppose the contrary, then by [9](2.13), $\left[H_{1}(C, \mathbb{Z}): H_{1}(S, \mathbb{Z})\right]=1$ and $C^{\prime}=p^{\star}(C)$ is a cycle with $2 s$ rational curves. Setting

$$
p^{\star}\left(D_{i}\right)=D_{i}^{\prime}+D_{n+i}^{\prime}, \quad p^{\star}\left(e_{i}\right)=e_{i}^{\prime}+e_{n+i}^{\prime}
$$

we have

$$
D_{0}^{\prime} D_{1}^{\prime}=\cdots=D_{s-2}^{\prime} D_{s-1}^{\prime}=D_{s-1}^{\prime} D_{n}^{\prime}=D_{n}^{\prime} D_{n+1}^{\prime}=\cdots=D_{n+s-1}^{\prime} D_{0}^{\prime}=1 .
$$

If we choose the numbering such that $D_{0}=-2 e_{1}-e_{I_{0}}$,

$$
D_{0}^{\prime}+D_{n}^{\prime}=-2\left(e_{1}^{\prime}+e_{n+1}^{\prime}\right)-e_{I_{0}}^{\prime}-e_{n+I_{0}}^{\prime} .
$$

Since $D_{0}^{\prime}$ and $D_{n}^{\prime}$ are of the same type and since they cannot be both of type $\mathbf{b}$ by lemma 2.7, both are of type a and there exists an index $i_{0}$ such that

$$
D_{0}^{\prime}=e_{0}^{\prime}-e_{n}^{\prime}-e_{1}^{\prime}-e_{n+1}^{\prime}-e_{I_{0}}^{\prime}, \quad D_{n}^{\prime}=e_{n}^{\prime}-e_{0}^{\prime}-e_{1}^{\prime}-e_{n+1}^{\prime}-e_{n+I_{0}}^{\prime} .
$$


By lemma 2.11,

$$
\sharp\left(C^{\prime}\right)-C^{\prime 2}=b_{2}\left(S^{\prime}\right)
$$

Besides

$$
\begin{aligned}
C^{\prime 2} & =\sum_{i=0}^{s-1}\left(D_{i}^{\prime 2}+D_{n+i}^{\prime 2}\right)+4 s=2\left(-4-\operatorname{Card}\left(I_{0}\right)+\sum_{i=1}^{s-1}\left(-1-\operatorname{Card}\left(I_{i}\right)\right)+2 s\right) \\
& =2\left(-3+s-\sum_{i=0}^{s-1} \operatorname{Card}\left(I_{i}\right)\right) .
\end{aligned}
$$

and by lemma 2.11,

$$
[0,2 n-1]=\{0, n, 1, n+1\} \cup \bigcup_{i=0}^{s-1}\left(I_{i} \cup n+I_{i}\right)
$$

therefore $[0, n-1]=\{0,1\} \cup \bigcup_{i=0}^{s-1} I_{i}$ and

$$
n=2+\sum_{i=0}^{s-1} \operatorname{Card}\left(I_{i}\right)
$$

These equalities yield

$$
\begin{aligned}
2 b_{2}(S) & =b_{2}\left(S^{\prime}\right)=\sharp\left(C^{\prime}\right)-C^{\prime 2}=2 s+2\left(3-s+\sum_{i=0}^{s-1} \operatorname{Card}\left(I_{i}\right)\right) \\
& =2\left(1+2+\sum_{i=0}^{s-1} \operatorname{Card}\left(I_{i}\right)\right)=2\left(1+b_{2}(S)\right),
\end{aligned}
$$

which is impossible.

Now, since $\left[H_{1}(C, \mathbb{Z}): H_{1}(S, \mathbb{Z})\right]=2$, a double covering $p: S^{\prime} \rightarrow S$ yields a surface $S^{\prime}$ with two cycles of rational curves, hence by [9], $S^{\prime}$ is an even Inoue-Hirzebruch surface and $S$ is an odd Inoue-Hirzebruch surface, in particular a Kato surface [3, thm 3.8.] By the explicit description of the self-intersection of the curves [3], the only possible curve $D_{0}$ of type $\mathbf{b}$ is $D_{0}=e_{0}-e_{[1, n-1]}-e_{0}-e_{1}=-2 e_{1}-e_{[2, n-1]}$, therefore $D_{0}^{2}=-(n+2)$ and $a(S)=\left(\overline{s_{n}}\right)$.

Remark 2. 13. In the previous computation subtraction $-e_{k}$ for a Kato surface means that we consider the strict transform by the blow-up by the exceptional curve $C_{k}$.

We notice that if $f: S^{\prime} \rightarrow S$ if a finite covering with $p$ sheets, then for $C^{\prime}=f^{\star}(C)$,

$$
\sharp\left(C^{\prime}\right)=p \sharp(C), \quad C^{\prime 2}=p C^{2}, \quad b_{2}\left(S^{\prime}\right)=p b_{2}(S) .
$$

Therefore the following result still holds for a rational curve with double point. We have proved:

Theorem 2. 14. Let $S$ be a minimal surface with a cycle $C=D_{0}+\cdots+D_{s-1}=-\left(e_{r}+\cdots+e_{n-1}\right)$ of $s \geq 1$ rational curves.

1) $S$ is an odd Inoue-Hirzebruch surface if and only if $\left[H_{1}(C, \mathbb{Z}): H_{1}(S, \mathbb{Z})\right]=2$. In this case $s=n, r=0$ and

$$
\sharp(C)-C^{2}=2 b_{2}(S) .
$$

2) If $H_{1}(C, \mathbb{Z})=H_{1}(S, \mathbb{Z})$, then $s=r$,

$$
\sharp(C)-C^{2}=b_{2}(S),
$$

all curves are of type a i.e. $D_{i}=e_{i}-e_{I_{i}}, i \notin I_{i}$, and $\left(I_{i}\right)_{0 \leq i \leq s-1}$ is a partition of $[0, n-1]$. 
We check these general results on Kato surfaces:

Examples 2. 15. Let $S$ be the Inoue-Hirzebruch surface such that

$$
a(S)=\left(\overline{s_{3} s_{1} s_{2}}\right)=(\overline{522342)}) .
$$
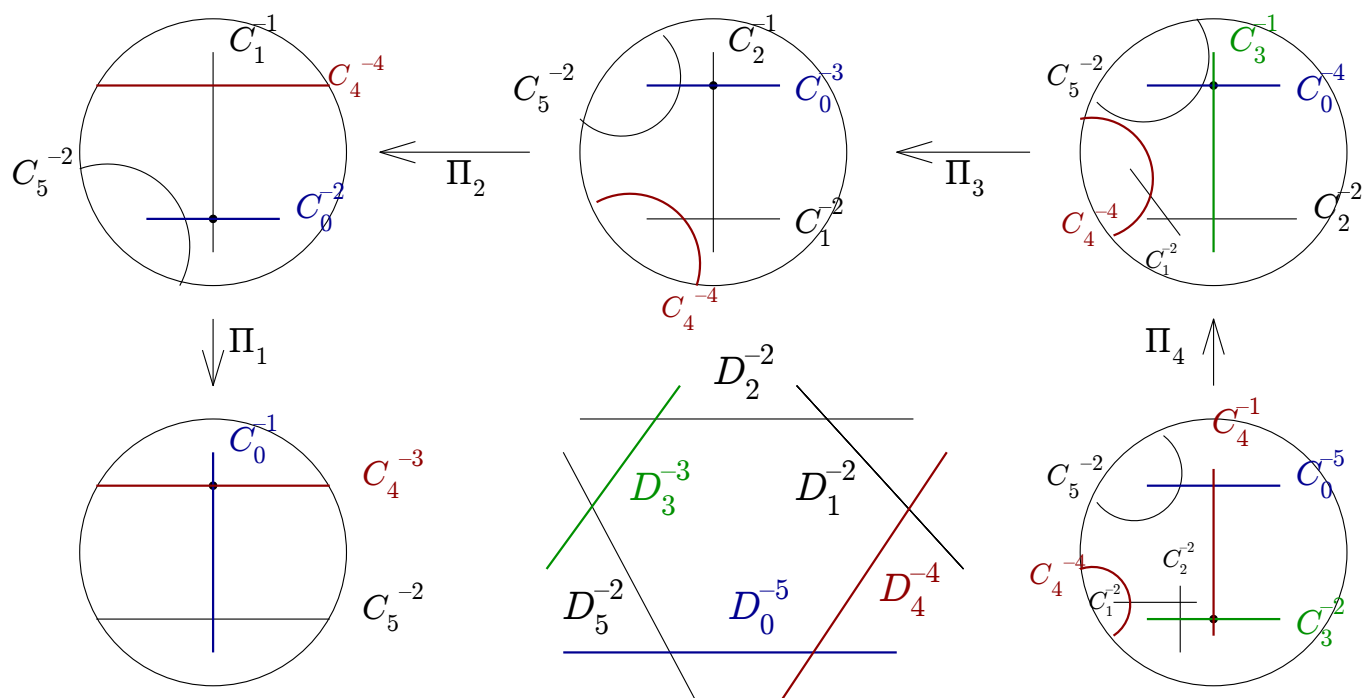

$\Pi_{4} \bigwedge$
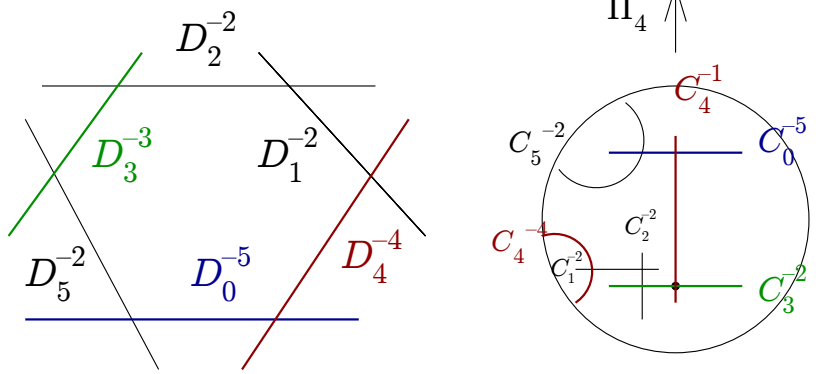

$\vee \Pi_{0}$

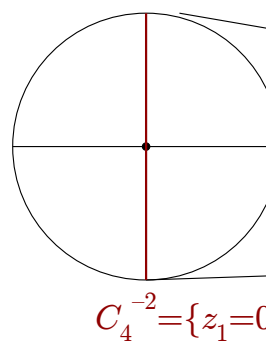

- blown-up point

$C_{5}^{-1}=\left\{z_{2}=0\right\}$
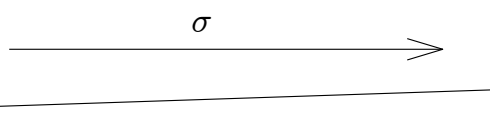

$\Pi_{5} \bigwedge$

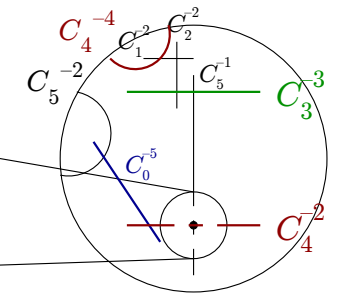

We denote by $D_{i}$ the curve induced in $S$ by $C_{i}$. Then

$$
\begin{array}{ll}
D_{0}=e_{0}-\left(e_{1}+e_{2}+e_{3}+e_{4}\right) & D_{1}=e_{1}-e_{2} \\
D_{2}=e_{2}-e_{3} & D_{3}=e_{3}-\left(e_{4}+e_{5}\right) \\
D_{4}=e_{4}-\left(e_{5}+e_{0}+e_{1}\right) & D_{5}=e_{5}-e_{0}
\end{array}
$$

Notice that there are two copies of each index in $\coprod_{i=0}^{5} I_{i}$.

Moving the blown-up point along $C_{0}$, we obtain a surface

$$
a(S)=\left(\overline{s_{3} s_{1} s_{1} r_{1}}\right)=(\overline{522332)}),
$$

with a regular sequence, hence with a branch (see [2]) 


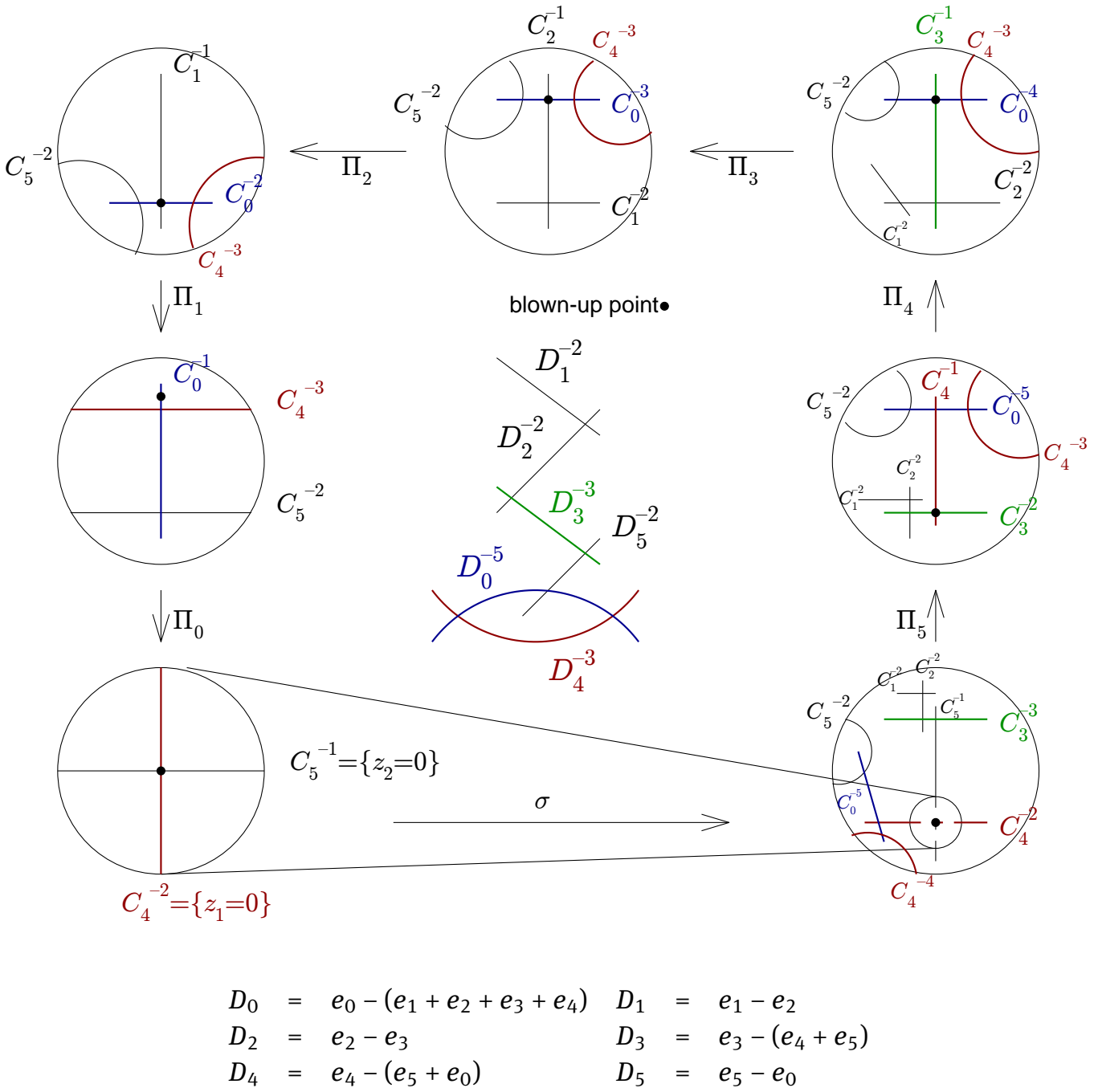

The cycle is $C=D_{0}+D_{4}, \sharp(C)-C^{2}=6=b_{2}(S), I_{0} \cap I_{4}=\emptyset$ and $I_{0} \cup I_{4}=[0,5]$.

It is well-known that any cycle may be realized in a even Inoue-Hirzebruch surface $S^{\prime}$, but in the following corollary we show that the second Betti number is the same:

Corollary 2. 16. Let $S$ be a minimal surface with a cycle $C=D_{0}+\cdots+D_{r-1} \sim-\left(e_{r}+\cdots+e_{n-1}\right)$ of $r \geq 1$ rational curves and let $n=b_{2}(S)$. Then there is an even Inoue-Hirzebruch surface $S^{\prime}$ with $b_{2}\left(S^{\prime}\right)=b_{2}(S)$ such that one of the two cycles, say $A=D_{0}^{\prime}+\cdots+D_{r-1}^{\prime}$ has the same intersection matrix.

Proof: We have by the previous theorem

$$
b_{2}\left(S^{\prime}\right)=-C^{\prime 2}+\sharp\left(C^{\prime}\right)=-C^{2}+\sharp(C)=b_{2}(S) .
$$

Corollary 2. 17. Let $S$ be a surface with a cycle $C$ of rational curves. When the curve numbered 0 is fixed, there is a numbering of the rational curves of the cycle and of the homological classes $e_{i}, i=0, \ldots, n-1$ such that (with $\alpha_{0}=0$ )

$$
C=D_{0}+\cdots+D_{\alpha_{i}}+\cdots+D_{\alpha_{s-1}}
$$


and in $H_{2}(S, \mathbb{Z})$, for $i=0, \ldots, s-1$,

$$
\begin{array}{cr}
D_{\alpha_{0}}=D_{0}=e_{0}-\left(e_{1}+\cdots+e_{\alpha_{1}}\right), & -D_{0}^{2}=\alpha_{1}+1, \\
D_{\alpha_{1}}=e_{\alpha_{1}}-\left(e_{\alpha_{1}+1}+\cdots+e_{\alpha_{2}}\right), & -D_{\alpha_{1}}^{2}=\alpha_{2}-\alpha_{1}+1, \\
\vdots & \vdots \\
D_{\alpha_{i}}=e_{\alpha_{i}}-\left(e_{\alpha_{i}+1}+\cdots+e_{\alpha_{i+1}}\right), & -D_{\alpha_{i}}^{2}=\alpha_{i+1}-\alpha_{i}+1 \\
\vdots & \vdots \\
D_{\alpha_{s-1}}=e_{\alpha_{s-1}}-\left(e_{\alpha_{s-1}+1}+\cdots+e_{n-1}+e_{0}\right), & -D_{\alpha_{s-1}}^{2}=n-\alpha_{s-1}+1 .
\end{array}
$$

Notice that

$$
D_{0} D_{\alpha_{1}}=\cdots=D_{\alpha_{i}} D_{\alpha_{i+1}}=\cdots=D_{\alpha_{s-1}} D_{0}=1 .
$$

In other words, denote by $\mathfrak{G} \subset[0, n-1]$ the subset of indices of rational curves in $C$, i.e.

$$
\mathfrak{G}=\left\{\alpha_{0}, \alpha_{1}, \ldots, \alpha_{s-1}\right\}
$$

then for $I=I_{C}=[0, n-1] \backslash \mathfrak{G}, C=-e_{I} \in H_{2}(S, \mathbb{Z})$. If there is a curve of type $\mathbf{b}, S$ is a Inoue-Hirzebruch surface by lemma 2.12, in particular $S$ is a Kato surface, therefore in the sequel we shall suppose that all curves are of type $\mathbf{a}$ and we shall write any curve of $C$ as

$$
D_{\alpha_{i}}=e_{\alpha_{i}}-e_{I_{\alpha_{i}}^{\prime}}-e_{\alpha_{i+1}}
$$

with $I_{\alpha_{i}}^{\prime}=I_{\alpha_{i}} \backslash\left\{\alpha_{i+1}\right\}$, then

$$
I_{C}=\bigcup_{i=0}^{s-1} I_{\alpha_{0}+\cdots+\alpha_{i}}^{\prime} .
$$

\section{3 (Co)homological class of the maximal divisor}

The following theorem shows that the class of the maximal divisor of a surface with a cycle of rational curves is of the same type as the class of a cycle. Moreover the maximal divisor looks like the one of a Kato surface: if there is one connected component there is a cycle and trees attached to different curves.

Lemma 3. 18. Let $S$ be a compact complex surface in class VII $I_{0}^{+}$endowed with a cycle $C=D_{\alpha_{0}}+\cdots+D_{\alpha_{i}}+\cdots+$ $D_{\alpha_{s-1}}$ of $s$ rational curves, $1 \leq s \leq n$, of class $-e_{I_{C}}, I_{C} \subset[0, n-1]$. Let $D_{\alpha_{i}}=e_{\alpha_{i}}-\left(e_{\alpha_{1}+1}+\cdots+e_{\alpha_{i+1}}\right)$ be the class of $D_{\alpha_{i}}$, i.e. $I_{C}=[0, n-1] \backslash\left\{\alpha_{0}, \ldots, \alpha_{s-1}\right\}$. Then there exists a deformation over the disc $\mathcal{S} \rightarrow \Delta$ which smoothes the point $D_{\alpha_{i}} \cap D_{\alpha_{i+1}}$, i.e. there is a flat families $\mathrm{C} \rightarrow \Delta$ such that

- $S_{0} \simeq S, S_{t}$ is not minimal, contains exactly one exceptional curve of the first kind of class $e_{\alpha_{i+1}}$,

- $C_{0} \simeq C, C_{t}=D_{\alpha_{0}}+\cdots+D_{\alpha_{i}}^{\prime}+D_{\alpha_{i+2}}+\cdots+D_{\alpha_{s-1}}, t \neq 0$, is of class $-e_{I_{C}}$ and $D_{\alpha_{i}}^{\prime}=D_{\alpha_{i}}+D_{\alpha_{i+1}}=e_{\alpha_{i}}-\left(e_{\alpha_{i}+1}+\right.$ $\left.\cdots+e_{\alpha_{i+1}-1}+e_{\alpha_{i+1}+1}+\cdots+e_{\alpha_{i+2}}\right)$.

Proof: By Karras [8], it is possible to smooth the singular point $D_{\alpha_{i}} \cap D_{\alpha_{i+1}}$ in order to obtain a cycle $C_{t}$ of rational curves (an elliptic curve if $s=1$ ) of the same class, in particular of the same self-intersection, but with one fewer rational curve. This local deformation can be globally realized by a deformation of surfaces. In fact, we have the exact sequence of sheaves

$$
0 \rightarrow \Theta_{S}(-\log C) \rightarrow \Theta_{S} \rightarrow J_{C} \rightarrow 0
$$

with $J_{C}=\Theta_{S} / \Theta_{S}(-\log C)$. Since for any curve $D_{\alpha_{i}}$ of $C, D_{\alpha_{i}}^{2} \leq-2, H^{0}\left(S, J_{C}\right)=0$. Besides with [9, lemma (4.3)] and $[10$, thm (1.3)], the long exact sequence of cohomology gives the exact sequence

$$
0 \rightarrow H^{1}\left(S, \Theta_{S}(-\log C)\right) \rightarrow H^{1}\left(S, \Theta_{S}\right) \rightarrow H^{1}\left(U, \Theta_{U}\right) \rightarrow 0
$$


for a strictly pseudoconvex neighbourhood $U$ of $C$.

We have in $H_{2}(S, \mathbb{Z})$

$$
D_{\alpha_{i}}^{\prime}=D_{\alpha_{i}}+D_{\alpha_{i+1}}
$$

By the formula of theorem 14, there is exactly one exceptional curve $E_{t}$ of the first kind of class the missing one in the expressions of classes of the curves in $C_{t}$, i.e. $E_{t}=e_{\alpha_{i+1}}$.

Theorem 3. 19. Let $S$ be a compact complex surface in class $V I I_{0}^{+}$endowed with exactly one cycle $C=D_{0}+$ $\cdots+D_{\alpha_{i}}+\cdots+D_{\alpha_{s-1}}$ of $s$ rational curves, $1 \leq s<n$, of class $-e_{I_{C}}$. Let $D=C+A$ be the maximal connected reduced divisor containing $C$ with $A \neq 0$. Let $D_{\alpha_{s}}, \ldots, D_{\alpha_{s+q-1}}$, be the irreducible components of $A$ numbered so that $\left(C \cup D_{\alpha_{s}} \cup \cdots \cup D_{\alpha_{s+k-1}}\right) \cap D_{\alpha_{s+k}} \neq \emptyset, k=0, \ldots, q-1$ (i.e. the unions remain connected). Then:

1. There is a family of subsets $\mathfrak{D}_{k} \subset[0, n-1], k \geq 0$, such that $\mathfrak{D}_{0}=I_{C}$ and

$$
D=C+A=-e_{\mathfrak{D}_{q-1}} \in H_{2}(S, \mathbb{Z}),
$$

2. Each connected component $A_{i}$ of $A$, called a tree, is a chain of rational curves,

3. Two different trees $A_{j}, A_{k}$, meet different curves of the cycle.

Proof: Since $p<n, S$ is not an odd Inoue-Hirzebruch surface and each class of curve has the form $e_{i}-e_{I_{i}}$. If $D_{\alpha_{s+k}}=e_{\alpha_{s+k}}-e_{I_{k}}$, we have for $k \geq 0$,

$$
\begin{aligned}
1 \leq D_{\alpha_{s+k}} \cdot\left(C+D_{\alpha_{s}}+\cdots+D_{\alpha_{s+k-1}}\right) & =-\left(e_{\alpha_{s+k}}-e_{I_{k}}\right) \cdot e_{\mathfrak{D}_{k-1}} \\
& =-e_{\alpha_{s+k}} \cdot e_{\mathfrak{D}_{k-1}}+e_{I_{k}} \cdot e_{\mathfrak{D}_{k-1}} \leq 1
\end{aligned}
$$

therefore

1. $\alpha_{s+k} \in \mathfrak{D}_{k-1}$,

2. $I_{k} \cap \mathfrak{D}_{k-1}=\emptyset$

3. there exists a unique index $i \in\{0, \ldots, s+k-1\}$ such that $D_{\alpha_{s+k}}$. $D_{\alpha_{i}}=1$ i.e. $D_{\alpha_{s+k}}$ meets only one curve at one point.

Finally

$$
C+D_{\alpha_{s}}+\cdots+D_{\alpha_{s+k}}=-e_{\mathfrak{D}_{k-1}}+\left(e_{\alpha_{s+k}}-e_{I_{k}}\right)=-e_{\mathfrak{D}_{k}}
$$

where $\mathfrak{D}_{k}=\left(\mathfrak{D}_{k-1} \backslash\left\{\alpha_{s+k}\right\}\right) \cup I_{k}$.

At each step $k \geq 0$, the indices of curves are in

$$
\left\{\alpha_{0}, \ldots, \alpha_{s-1}, \alpha_{s}, \ldots, \alpha_{s+k-1}\right\}
$$

and $D_{\alpha_{s+k}}$ meets a curve $D_{\alpha_{i}}$ of the cycle without any other tree or the top of a tree. In fact, suppose the contrary:

- If $D_{\alpha_{s+k_{1}}}=e_{\alpha_{s+k_{1}}}-e_{I_{s+k_{1}}}$ and $D_{\alpha_{s+k_{2}}}$ meet the same curve in the cycle, say $D_{\alpha_{0}}$. Then we have for $D_{\alpha_{s+k_{1}}}$ the system

$$
\begin{aligned}
& 1=D_{\alpha_{0}} . D_{\alpha_{s+k_{1}}}=\left[e_{\alpha_{0}}-\left(e_{\alpha_{0}+1}+\cdots+e_{\alpha_{1}}\right] .\left[e_{\alpha_{s+k_{1}}}-e_{I_{s+k_{1}}}\right]\right. \\
& \left.0=D_{\alpha_{1}} \cdot D_{\alpha_{s+k_{1}}}=\left[e_{\alpha_{1}}-\left(e_{\alpha_{1}+1}+\cdots+e_{\alpha_{2}}\right)\right] .\left[e_{\alpha_{s+k_{1}}}-e_{I_{s+k_{1}}}\right)\right] \\
& \text {.. } \quad \ldots \\
& \left.0=D_{\alpha_{j}} . D_{\alpha_{s+k_{1}}}=\left[e_{\alpha_{j}}-\left(e_{\alpha_{j}+1}+\cdots+e_{\alpha_{j+1}}\right)\right] .\left[e_{\alpha_{s+k_{1}}}-e_{I_{s+k_{1}}}\right)\right]
\end{aligned}
$$

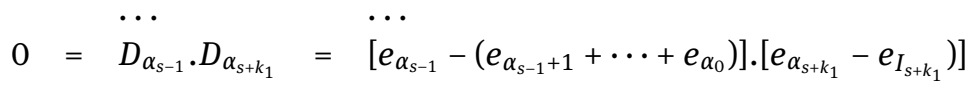

Recall that if $D^{\prime}=e_{i^{\prime}}-e_{I^{\prime}}$ and $D^{\prime \prime}=e_{i^{\prime \prime}}-e_{I^{\prime \prime}}$ are distinct curves, then $i^{\prime} \neq i^{\prime \prime}$. 
1. If $\alpha_{s+k_{1}} \in\left\{\alpha_{0}+1, \ldots, \alpha_{1}\right\}$ the system is equivalent to

$$
\begin{aligned}
0= & -e_{\alpha_{0}} \cdot e_{I_{s+k_{1}}}+\left(e_{\alpha_{0}+1}+\cdots+e_{\alpha_{1}}\right) \cdot e_{I_{s+k_{1}}} \\
0= & -e_{\alpha_{1}} \cdot e_{I_{s+k_{1}}}+\left(e_{\alpha_{1}+1}+\cdots+e_{\alpha_{2}}\right) e_{I_{s+k_{1}}} \\
& \cdots \\
0= & -e_{\alpha_{s-1}} \cdot e_{I_{s+k_{1}}}+\left(e_{\alpha_{s-1}+1}+\cdots+e_{\alpha_{0}}\right) e_{I_{s+k_{1}}}
\end{aligned}
$$

We have $I_{s+k_{1}} \subset\left\{\alpha_{0}, \ldots, \alpha_{s-1}\right\}$ (recall condition (2)), then there are two cases

- if $\alpha_{0} \notin I_{s+k_{1}}$ then $\alpha_{1} \notin I_{s+k_{1}}, \ldots, \alpha_{s-1} \notin I_{s+k_{1}}$ and $I_{s+k_{1}}=\emptyset$ which is impossible. Contrarily

- if $\alpha_{0} \in I_{s+k_{1}}$, then the equations imply $\alpha_{1} \in I_{s+k_{1}}, \ldots, \alpha_{s-1} \in I_{s+k_{1}}$ and

$$
I_{s+k_{1}}=\left\{\alpha_{0}, \ldots, \alpha_{s-1}\right\}, \quad \text { Card } I_{s+k_{1}}=s \geq 1 .
$$

2. If $\alpha_{s+k_{1}} \in\left\{\alpha_{j}+1, \ldots, \alpha_{j+1}\right\}, j>0$, the system is equivalent to

$$
\begin{aligned}
1= & -e_{\alpha_{0}} \cdot e_{I_{k_{1}}}+\left(e_{\alpha_{0}+1}+\cdots+e_{\alpha_{1}}\right) \cdot e_{I_{s+k_{1}}} \\
0= & -e_{\alpha_{1}} \cdot e_{I_{k_{1}}}+\left(e_{\alpha_{1}+1}+\cdots+e_{\alpha_{2}}\right) \cdot e_{I_{s+k_{1}}} \\
& \cdots \\
0= & -e_{\alpha_{j}} \cdot e_{I_{k_{1}}}+1+\left(e_{\alpha_{j}+1}+\cdots+e_{\alpha_{j+1}}\right) \cdot e_{I_{s+k_{1}}} \\
0= & -e_{\alpha_{j+1}} \cdot e_{I_{k_{1}}}+\left(e_{\alpha_{j+1}+1}+\cdots+e_{\alpha_{j+2}}\right) \cdot e_{I_{s+k_{1}}} \\
& \cdots \\
0 & -e_{\alpha_{s-1}} \cdot e_{I_{k_{1}}}+\left(e_{\alpha_{s-1}+1}+\cdots+e_{\alpha_{0}}\right) e_{I_{s+k_{1}}}
\end{aligned}
$$

and since $I_{s+k_{1}} \subset\left\{\alpha_{0}, \ldots, \alpha_{s-1}\right\}$

$$
I_{s+k_{1}}=\left\{\alpha_{j+1}, \ldots, \alpha_{s-1}, \alpha_{0}\right\}, \quad \operatorname{Card} I_{s+k_{1}}=s-j \geq 1 .
$$

We have the same conditions for $D_{\alpha_{s+k_{2}}}$ therefore an easy calculation shows that $D_{\alpha_{s+k_{1}}} \cdot D_{\alpha_{s+k_{2}}}<0$ which is impossible.

- We have to check now that each connected component $A_{i}$ of $A$ is a chain of rational curves. We prove that by induction on the number of the rational curves of the cycle.

If $\boldsymbol{p}=\mathbf{1}$, we can number the Donaldson classes such that

$$
D_{0}=e_{0}-\left(e_{1}+\cdots+e_{n-1}+e_{0}\right)=-\left(e_{1}+\cdots+e_{n-1}\right) .
$$

If a curve $D_{1}=e_{k}-e_{I_{1}}$ meets $D_{0}$,

$$
\begin{aligned}
1 \leq D_{0} \cdot D_{1} & =-\left(e_{1}+\cdots+e_{n-1}\right) \cdot\left(e_{k}-e_{I_{1}}\right) \\
& =-\left(e_{1}+\cdots+e_{n-1}\right) \cdot e_{k}+\left(e_{1}+\cdots+e_{n-1}\right) \cdot e_{I_{1}} \leq 1
\end{aligned}
$$

therefore $1 \leq k \leq n-1$ and $I_{1} \cap\{1, \ldots, n-1\}=\emptyset$. It means that $I_{1}=\{0\}$. If another curve $D^{\prime}=e_{k^{\prime}}-e_{I^{\prime}}$ meets $D_{0}$ we have also $I^{\prime}=\{0\}$ and $D_{1} \cdot D^{\prime}=\left(e_{k}-e_{0}\right) \cdot\left(e_{k^{\prime}}-e_{0}\right)=-1$ which is impossible. We choose the numbering such that $k=1$ and $D_{1}=e_{1}-e_{0}$. We have $D_{0}+D_{1}=-\left(e_{0}+e_{2}+\cdots+e_{n-1}\right)$. Let $D_{2}=e_{l}-e_{I_{2}}$ with a non-empty intersection with $D_{0}+D_{1}$. We have

$$
1 \leq\left(D_{0}+D_{1}\right) \cdot D_{2}=-\left(e_{0}+e_{2}+\cdots+e_{n-1}\right) \cdot\left(e_{l}-e_{I_{2}}\right) \leq 1 .
$$

Hence $l \in\{0,2, \ldots, n-1\}$ and $I_{2}=\{1\}$, i.e. if numbering is choosen such that $l=2, D_{2}=e_{2}-e_{1}$. By induction we obtain a chain.

If $\boldsymbol{p}>\mathbf{1}$ Suppose that we have the result for cycles of $p-1 \geq 1$ curves, and let $C=D_{\alpha_{0}}+\cdots+D_{\alpha_{p}-1}$ be a cycle of $p$ curves. By lemma 18, there is a deformation which smoothes a singular point of the cycle, say $D_{\alpha_{0}} \cap D_{\alpha_{1}}$, then self-intersection does not change and the number of curves decreases by one, therefore the deformed surface in not minimal (recall the formula $b_{2}(S)=-C^{2}+\sharp(C)$ for minimal surfaces) with exactly one exceptional curve of the first kind of class $e_{\alpha_{1}}$. If the new rational curve $D_{0}^{\prime}$, of the same class as $D_{\alpha_{0}}+D_{\alpha_{1}}$, is intersected by one connected component, all connected components are chains by the induction hypothesis. If two connected components meet $D_{0}^{\prime}$, then by the induction hypothesis one of the two is an exceptional divisor. Since there is only one exceptional curve of the first kind this component is also a chain. 
Remark 3. 20. It is difficult to detect more curves than those in the cycle, since the expressions of $C$ and of the maximal divisor $\mathrm{D}=\mathrm{C}+\mathrm{A}$ in $\mathrm{H}_{2}(\mathrm{~S}, \mathbb{Z})$

$$
C=-e_{[0, n-1] \backslash \mathfrak{G}}, \quad D=\Gamma+A=-e_{\mathfrak{D}}
$$

have the same type, i.e. the coefficients are all equal to 0 or -1 ; they have also same genus $p_{a}(C)=p_{a}(C+A)=1$, where the arithmetic genus $p_{a}$ of a divisor $\Delta$ is defined by $p_{a}(\Delta)=1+\frac{1}{2}\left(K \Delta+\Delta^{2}\right)$.

For example, if $b_{2}=2$ the class of a cycle $C$ does not determine the maximal divisor: it may be either a cycle $C=-e_{1}$ with one curve and another cycle $C^{\prime}=-e_{0}$ or a cycle $C=-e_{1}$ with a tree $A=e_{1}-e_{0}$.

\section{Connected components of the maximal divisor}

Lemma 4. 21. Let $S$ be a surface in class VII ${ }_{0}^{+}$containing a cycle $C$ of rational curves with $n=b_{2}(S) \geq 1$. We suppose that $S$ is not an odd Inoue-Hirzebruch surface. Let $H$ be a reduced divisor whose support is connected and simply connected. Then there is an integer $k \in\{0, \ldots, n-1\}$ and a subset $K \subset\{0, \ldots, n-1\}$, with $k \notin K$, such that in $\mathrm{H}_{2}(S, \mathbb{Z})$

$$
H=e_{k}-e_{K}
$$

Proof: Since the surface is not an odd Inoue-Hirzebruch surface the class of any rational curve is of the type $e_{i}-e_{I}$. We prove the result by induction on the number of irreducible components with the same arguments already used in theorem 19.

Theorem 4.22. Let $S$ be a surface in class VII $I_{0}^{+}$with $b_{2}(S)=n$, containing a cycle $C=D_{\alpha_{0}}+\cdots+D_{\alpha_{s-1}}$ of $s$ rational curves and $D=C+A$ the connected component of the cycle. If there is another connected component of curves $C^{\prime} \neq 0$, then $C^{\prime}$ is a cycle of rational curves, $A=0$ and $S$ is a Inoue-Hirzebruch surface.

Proof: Let $I \subset\{0, \ldots, n-1\}$ such that $C=-e_{I}$. By Corollary 17, indices of the curves of $C$ belong to $\left\{\alpha_{0}, \ldots, \alpha_{s-1}\right\}$ and $I=[0, n-1] \backslash\left\{\alpha_{0}, \ldots, \alpha_{s-1}\right\}$. Suppose that $C^{\prime}$ does not contain a cycle of rational curves. Then $C^{\prime}$ is simply connected. Since $C^{\prime} . D=0$, Lemma 21 , shows that $C^{\prime}=e_{k}-e_{K}$, with $k \in I, k \notin K$, and $K \cap I=\{l\}$. Similarly if $A_{i}$ is a tree, since $A_{i} . C=1, A_{i}=e_{j_{i}}-e_{J_{i}}$ with $j_{i} \in I$ and $J_{i} \cap I=\emptyset$. Moreover since two branches $A_{i}=e_{j_{i}}-e_{J_{i}}$ and $A_{i^{\prime}}=e_{j_{i^{\prime}}}-e_{J_{i^{\prime}}}$ do not meet $J_{i} \cap J_{i^{\prime}}=\emptyset$. We apply now theorem (1.4) of [10] with $H=A+C^{\prime}$ in order to obtain a deformation $\mathcal{S} \rightarrow \Delta^{S}$ with flat families $\mathcal{C}$ and $\mathcal{H}$ where $H_{t}=H$, and $\mathcal{C} \rightarrow \Delta^{S}$ is the versal deformation of $C$, in particular generically $C_{t}$ is an elliptic curve of class $-e_{I}$ and $S_{t}$ contains exceptional divisors of the first kind $E_{\alpha_{0}}=e_{\alpha_{0}}, \ldots, E_{\alpha_{s-1}}=e_{\alpha_{s-1}}$. The only minimal surfaces in class VII ${ }_{0}^{+}$with an elliptic curve are Hopf surfaces and Inoue surfaces. Therefore, generically, $S_{t}$ is a blown-up Inoue surface or a blown-up Hopf surface with minimal model $S_{t}^{\prime}$ of second Betti number $0 \leq b_{2}\left(S_{t}^{\prime}\right) \leq n-s$. There is a disc $\Delta$ over which $C_{t}$ is an elliptic curve, blown-up by exceptional divisors of the first kind $E_{i}=e_{i}, i \in I^{\prime}, I^{\prime} \subset I$, exceptional divisors $E_{\alpha_{j}}, 0 \leq j \leq s-1$ and $C^{\prime}=e_{k}-e_{K}$. If $I^{\prime} \neq I, S_{t}$ is a blown-up Inoue surface and contains also a cycle

$$
\Gamma_{t}=\sum_{i \in I \backslash I^{\prime}} D_{i},
$$

with $D_{i}=e_{i}-e_{i+1}$ (when $I \backslash I^{\prime}$ is properly numbered modulo Card $(I)$ - Card $\left(I^{\prime}\right)$ ). Since $k \in I$ there are two cases:

- $k \in I^{\prime}$, then $C^{\prime} \cdot E_{k}=\left(e_{k}-e_{K}\right) \cdot e_{k}=-1$ impossible,

- $k \in I \backslash I^{\prime}$ then $C^{\prime} \cdot D_{k}=\left(e_{k}-e_{K}\right) \cdot\left(e_{k}-e_{k+1}\right)=-1+e_{K} \cdot e_{k+1}<0$ impossible

If $I^{\prime}=I$ we are in the first case.

In any case we obtain a contradiction. 
Now, $C^{\prime}$ contains a cycle of rational curves, we have two cycles, we conclude by a theorem of Nakamura [9, Thm 8.1] that $S$ is a Inoue-Hirzebruch surface, $C^{\prime}$ is a cycle and $A=0$.

\section{Application: Surfaces with twisted logarithmic forms}

Lemma 5.23. Let $S$ be a surface in class VII+ which is not a Enoki surface. If there exists a non-trivial twisted logarithmic 1-form with polar set $D$,

$$
0 \neq \omega \in H^{0}\left(S, \Omega^{1}(\log D) \otimes \mathcal{L}\right)
$$

with $\mathcal{L} \in H^{1}\left(S, \mathbb{C}^{\star}\right)$, then $D \neq 0$ and each connected component of $D$ contains a cycle of rational curves. In particular, there is at most two connected components and in this case $S$ is a Inoue-Hirzebruch surface.

Proof: By [1, Thm 5.6] there exists a line bundle $\mathcal{L} \in \operatorname{Pic}^{0}(S)$ such that $H^{1}\left(S, \Omega^{1} \otimes \mathcal{L}\right) \neq 0$ only if $S$ is a Enoki surface, therefore $D \neq 0$. Let $D^{\prime}$ be any connected component of the polar set $D$ of $\omega$, then the intersection matrix is negative definite by [7]. Let a spc neighbourhood $V^{\prime}$ of $D^{\prime}$ sufficiently small to be contractible onto a Stein space $V$ with an isolated singular normal point $x \in V$. Let $\pi: V^{\prime} \rightarrow V$ be this contraction, $D^{\prime}=\pi^{-1}(x)$ be the exceptional divisor. Let $U=V \backslash\{x\}$. If $D^{\prime}$ does not contain a cycle, $D^{\prime}$ is simply connected then the restriction of $\mathcal{L}$ to $V^{\prime}$ is trivial . By the theorem of Steenbrinck-Van Straten [11, Cor. 1.4] the mapping induced by the differentiation

$$
d: H^{0}\left(U, \Omega_{U}^{1}\right) / H^{0}\left(V^{\prime}, \Omega_{V^{\prime}}^{1}\right) \rightarrow H^{0}\left(U, \Omega_{U}^{2}\right) / H^{0}\left(V^{\prime}, \Omega_{V^{\prime}}^{2}\left(\log D^{\prime}\right)\right)
$$

is injective, which gives a contradiction if there is no cycle. If there is a cycle and another connected component of rational curves then there are two cycles by theorem 3.22.

Proposition 5. 24. Let $S$ be a surface in class VII $0_{0}^{+}$. If there exists a non-trivial twisted logarithmic 1-form with polar set $D$,

$$
0 \neq \omega \in H^{0}\left(S, \Omega^{1}(\log D) \otimes \mathcal{L}\right)
$$

with $\mathcal{L} \in H^{1}\left(S, \mathbb{C}^{\star}\right)$, then the vanishing divisor of $\omega$ is trivial.

Proof: If $S$ is a Enoki surface then any twisted logarithmic or holomorphic 1-form does not vanish. If $S$ is not a Enoki surface and there is a divisor $A$ where $\omega$ vanishes, $A \cap D=\emptyset$ and there are two connected components of curves, therefore $S$ would be a Inoue-Hirzebruch surface by theorem 3.22. However on these surfaces $\omega$ does not vanishes at all.

Conflict of interest: Author states no conflict of interest.

\section{References}

[1] V. Apostolov, \& G. Dloussky, On the Lee classes of locally conformally symplectic complex surfaces. J. of symplectic geometry $16 n^{0} 4$, pp 931-958 (2018).

[2] G. Dloussky, Structure des surfaces de Kato. Mémoire de la S.M.F112.n 14 (1984).

[3] G. Dloussky, Une construction élémentaire des surfaces d'Inoue-Hirzebruch. Math. Ann. 280, (1988), 663-682.

[4] G. Dloussky, On surfaces of class $\mathrm{VII}_{0}^{+}$with numerically anticanonical divisor. Am. J. Math. 128, 639-670 (2006)

[5] G. Dloussky, K. Oeljeklaus, M. Toma, Class VII 0 surfaces with $b_{2}$ curves. Tohoku Math. J. 55 (2003), 283-309

[6] S.K. Donaldson, The orientation of Yang-Mills moduli space and 4-manifolds topology. J. Differential Geometry 26 (1987) 397-428.

[7] I. Enoki, Surfaces of class VII 0 with curves. Tôhoku Math. J. 33, (1981), 453-492.

[8] U. Karras, Deformations of cusp singularities, Proc. of Symp. in Pure Math. 30, 1977, p37-43. 
[9] I. Nakamura, On surfaces of class $\mathrm{VII}_{0}$ with curves. Invent. Math. 78, (1984), 393-443.

[10] I. Nakamura, On surfaces of class VII 0 with curves II.Tohoku Math. J. 42 (1990), 475-516.

[11] J. Steenbrink \& D. van Straten, Extendability of holomorphic differential forms near isolated hypersurface singularities Abh. Math. Sem. Univ. Hamburg 55, 97-110 (1985)

[12] A. Teleman, Donaldson theory on non-Kählerian surfaces and class VII surfaces with $b_{2}=1$, Invent. math. 162, 493-521 (2005)

[13] A. Teleman, Gauge theoretical methods in the classification of non-Kählerian surfaces, Algebraic topology - old and new old and new, Banach Center Publ . 85 (2009) 109-120.

[14] A. Teleman, Instantons and curves on class VII surfaces, Annals of Math. (2) 172 (2010), $n^{\circ} 3,1749-1804$. 